\title{
Selective assessment of judgmental inconsistencies in pairwise comparisons for group decision rating
}

\author{
Frej Limayem ${ }^{\mathrm{a}}$, Bernard Yannou ${ }^{\mathrm{b}}$ * \\ ${ }^{a, b}$ Laboratoire Génie Industriel, Ecole Centrale Paris, Grande Voie des Vignes, 92295, \\ Châtenay-Malabry, France
}

\begin{abstract}
Pairwise comparison methods are convenient procedures for providing a sound weight vector from a set of binary comparisons between elements to be rated. In such procedures, each decision maker is asked to separately consider pairs of elements which are not necessarily independent from each other. For this reason the votes collected are liable to contain inconsistencies. In this paper we are providing a selective indicator that focuses on the inconsistencies the decision group is willing to correct in conformity with its vote strategy.
\end{abstract}

Keywords: pairwise comparison, decision making, logarithmic least squares regression, inconsistency.

\section{Introduction}

Rating a set of $n$ elements $\left(e_{1}, \ldots, e_{n}\right)$ can be a demanding task even when a unique criterion is considered. A convenient class of methods called pairwise comparison methods (PCM) notably simplifies the problem by focusing the attention on pairs of elements to be compared under a given property or criterion. The comparison matrix (see figure 1) represents all possible combinations.

More generally, the theory of preference ${ }^{1}$ representation addresses such mappings from local comparisons to global ratings/rankings. It proposes a general multi-criteria decision-making framework where variable grades of preference are considered in combination with importance coefficients and even hierarchical structures on the criteria in order to assess, from a comprehensive point of view, the corresponding system of order on the elements set [17].

The different approaches articulate on common components [3]:

- elements (alternatives or sub-objectives) to be compared;

- criteria to assess them;

- aggregation methods;

- criteria-based evaluations of elements or alternatives;

- inter-criteria information such as for example weights.

\footnotetext{
*E-mail address: <frej_limayem $><$ Bernard_yannou $>@$ hotmail.com

${ }^{1}$ The intangible nature of preference induces different approaches among existing PCM schools. For example, the Multi-Attribute Value Theory (MAVT) measures preferences through an origin/unit scale $(p=\alpha \times u+\beta)$ by comparing differences of preference of the form $\left(p_{i}-p_{j}\right) /\left(p_{k}-p_{h}\right)$, while the AHP school directly compares preferences of the form $p_{i} / p_{j}$, assuming more intuitive the existence of an absolute origin for preference $(\beta=0)$ [2]. Despite the criticism it is subject to, the later hypothesis is adopted in an important part of the literature and numerous applications [20].
} 
In group decision making, the three first points are generally common to all the participants. The group can enclose several roles (ex: decision makers, facilitators, experts), different profiles (degree of power/influence, degree of expertise, etc.) and several points of view ${ }^{2}$. Three generic modes are proposed in the literature to deal with the differences:

- consensual merging of views through a causal analysis of their divergence in order to reduce it;

- finding a compromise through a vote or the calculation of a representative value;

- comparing independent results in order to negotiate a consensual view without necessarily reducing the underlying divergences [3].

A vote strategy can be perceived as a particular mapping between the group structure and the five components above.

This paper considers a mono-criteria decision making framework where pairwise comparisons are associated, through a given ratio scale ${ }^{3}$, to numerical values $c_{i j}$ in order to estimate the ratio of element weights $w_{i} / w_{j}$ (note 1 ).

Chronologically, the first applications [11] addressed basic comparison matrices with exactly one deterministic (precise) opinion per comparison. In parallel to the introduction of imprecision and uncertainty (ex: $[4 ; 10])$, pairwise comparison methods have lately evolved to tackle abstentions or situations where the presence of several decision makers (DMs) can lead to more than one opinion per binary comparison. The concept of comparison matrix can then be extended to a comparison cube.

Let $c_{i h x}$ represent the opinion of the DM of index $x$ when estimating the importance ratio of $e_{i}$ over $e_{h}$. Since there are $n$ unknown weights and up to $d \times n^{2}$ different equations (if each DM expresses $n^{2}$ comparisons), the system is likely to be overconstrained with no a priori best set of weights. This is why different optimization logics may be considered.

This redundancy generally results in a cardinal intransitivity (if at least one of the following equalities do not hold: $\left.c_{i j x} c_{j h y} \cdot c_{h i z}=1 ; i, j, h=1,2, \ldots, \mathrm{n} ; x, y, z=1,2, \ldots, d\right)^{4}$. An even more severe form (because illogical), called ordinal intransitivity, occurs in presence of cyclic binary comparisons (ex: $c_{i h x}>1$ and $c_{h j y}>1$ but $c_{i j z}<1 ; i, j, h=1,2, \ldots, n$; $x, y, z=1,2, \ldots, d)$. When DMs reduce to a unique voter, the so called intransitivity is generally perceived as inconsistency especially for the ordinal form.

This is less true for group decision making where opinions' redundancy occurs naturally. In such a context, various vote strategies can be considered, in which intransitivity is not synonym of inconsistency and in which consistency indicators should take into account both the vote strategy and procedure. Let us mention the case

\footnotetext{
${ }^{2}$ Decision makers can for example be perceived as a non disjoint set of voting entities (of one or several DMs), providing each a uniform point of view on a subset of the criteria-based evaluations and having homogenous attributes (ex: weight, access to others votes, etc.) [13].

${ }^{3}$ Ratio scales associate numerical values to linguistic variables expressing different orders of importance/preference. Different scales are used in the literature according to the nature of the elements to be rated (ex: objects or criteria) [16] or the type of aggregation function in which the weights are going to be used (ex: additive or multiplicative). As illustrated in [20], ratio scales often distort the quality of the results (ex: min and max boundaries for the weights). Two examples of ratio scales are given in the remainder of this paper, at section 5 .

${ }^{4}$ Intransitivity is easy to observe on paths of length 2 (ex: $c_{i j} c_{j i} \neq 1$ ) or 1 (when $\left.c_{i i} \neq 1\right)$. As mentioned in [21], intransitivity can also be pointed out through indirect paths of length $l>3$ (ex: of the form $c_{i j} c_{j k} c_{k h}$ $c_{h i} \neq 1$, for $l=4$ ). In general the longer the path the more excusable the intransitivity since more difficult to trace for DMs.
} 
where the decision group is not necessarily seeking a unique point of view shared by all the participants but a solution that could be representative of divergent opinions, for instance the barycenter of group's judgments. In such a case, the violation of the transitivity condition should be allowed for opinions expressed by different DMs and no corrective actions ${ }^{5}$ should be initiated towards the outliers. Beyond the redundancy of opinions within the group, other properties can be questioned at DMs' scale such as for example the reciprocity of the comparison matrix, i.e. the fact that symmetrical opinions are inverse of each other; one also speaks about reciprocal opinions. Indeed, in a blind test involving sensorial evaluations (ex: beverage/food tasting or perfume smelling), the ordering in which each of the two elements is considered can significantly influence the appreciation. In such a case it is quite normal to expect from a same DM non reciprocal opinions (between $c_{i j k}$ and $c_{j i k}$ ) and thus tolerate the corresponding part of inconsistency.

More generally, at different voting levels (ex: isolate DMs versus DMs' clusters) ${ }^{2}$, consistency measures should be able to reflect the conformity of the decision making process to the vote strategy. Among the existing indicators, just a few are able to tackle such nuances.

This paper attempts to provide a more selective approach that helps in focusing on inconsistencies that the decision group is willing to correct. As it opens interesting perspectives, it also highlights some limits of this concept of « selective assessment of inconsistencies ».

In the following section, a brief literature review sets the focus on indicators that have addressed the issue of group decision making. Next, section 3 introduces a new consistency indicator based on a logarithmic regression approach. The perspective of a selective assessment of inconsistency is then developed in section 4. Before concluding, an illustrative example is presented in section 5.

\section{Existing indicators}

Table 1 classifies some of the indicators published in the literature according to two criteria: their sensitivity to rating scales and the underlying pairwise comparison method (EV for Eigenvector, GM for geometric mean, MP for mathematical programming and LLSR for logarithmic least squares regression)

\footnotetext{
${ }^{5}$ Inconsistency measures are usually associated to threshold values ([10], [18], [12]) and/or followed by corrective actions that can take the form of iterative feedback loops between individuals consistency [5] and overall group's consistency ([13], [15]).
} 


\begin{tabular}{|c|c|c|c|c|c|}
\cline { 2 - 5 } \multicolumn{1}{c|}{} & $\begin{array}{c}\text { No specific } \\
\text { method }\end{array}$ & EV & GM & MP & LLSR \\
\hline $\begin{array}{c}\text { Scale } \\
\text { dependent }\end{array}$ & $\begin{array}{c}\text { Golden and } \\
\text { Wang's } \\
\text { indicator [12] }\end{array}$ & $\begin{array}{c}\text { Saaty's } \\
\text { indicator [19] }\end{array}$ & $\begin{array}{c}\text { Takeda's indicator } \\
\text { [21] }\end{array}$ & $\begin{array}{c}\text { Bryson and } \\
\text { Joseph's } \\
\text { indicator [5] }\end{array}$ & $\begin{array}{c}\text { Crawford and } \\
\text { Williams' } \\
\text { indicator [7] }\end{array}$ \\
\hline $\begin{array}{c}\text { Scale } \\
\text { independent }\end{array}$ & $\begin{array}{c}\text { Salo's } \\
\text { indicator [20] }\end{array}$ & \multicolumn{4}{|c}{} \\
\cline { 1 - 5 }
\end{tabular}

Tab. 1: Some indicators published in the literature

In the remainder of this section one reviews more in detail indicators that have addressed the issue of group decision making.

\subsection{Saaty's indicator}

Saaty's consistency indicator is based on the well known eigenvector method [18] and requires, in addition to exactly one opinion per comparison, a reciprocal comparison matrix, where all symmetrical binary comparisons are inverse of each other (i.e. $\left.c_{i j}=1 / c_{j i} ; i, j=1,2, \ldots, n\right)$. In the formula hereafter, the largest eigenvalue $\lambda_{\max }$ equals $n$ for perfectly consistent comparison matrices $($ C.I. saaty $=0)$ and take large values ${ }^{6}$ for very inconsistent ones.

$$
\mid \begin{array}{l:l}
\text { C.I. Saaty } & =\frac{\lambda_{\max }-n}{n-1} \\
\text { with } & \lambda_{\max } \text { :largest eigen value associated to the comparison matrix, } \\
& n: \text { number of elements to be weighted. }
\end{array}
$$

When exactly one opinion is required, four vote strategies seem to prevail for the group [6]: preliminary consensus on each entry, vote compromise on each entry, average of the individual judgments on each entry or weighted average of the individual judgments on each entry. In such a context an alternative form called relative departure from consistency [19] has been used to estimate the relative divergence between a given decision maker and the group (formula 2).

$$
\begin{aligned}
& \text { C.I. Saaty, group }_{\text {max }}=\frac{\lambda_{\max }-n}{n}=\frac{1}{\mathrm{n}^{2}}\left(\sum_{i, j=1}^{n} \overline{c_{i j}} \frac{w_{j}}{w_{i}}-\mathrm{n}^{2}\right) \text {, with } \overline{c_{i j}}=\left(\prod_{k=1}^{d} c_{i j k}\right)^{1 / d} . \\
& \text { C.I. Saaty, } D M_{k}=\frac{\lambda_{\max }-n}{n}=\frac{1}{\mathrm{n}^{2}}\left(\sum_{i, j=1}^{n} c_{i j k} \frac{w_{j}^{k}}{w_{i}^{k}}-\mathrm{n}^{2}\right) \text {, with } \begin{array}{l}
w_{i}^{k}, i=1,2, \ldots, n, \text { the weights deduced } \\
\text { from } \mathrm{DM}_{k} \text { 's comparison matrix. }
\end{array} \\
& \text { C.I. Saaty,DM } \text { / group }=\frac{1}{\mathrm{n}^{2}}\left(\sum_{i, j=1}^{n} c_{i j k} \frac{w_{j}}{w_{i}}-\mathrm{n}^{2}\right): \begin{array}{l}
\text { relative departure of the } k^{\text {th }} \text { individual's } \\
\text { judgements from those of the groupe. }
\end{array}
\end{aligned}
$$

\footnotetext{
${ }^{6}$ In order to rescale his indicator between 0 and 1 , Saaty proposes a linear transformation dividing his indicator by the quantity $n(g-1) /(n-1)$, with $g$ the number of values composing the notation scale. He has also considered the sensitivity of his indicator to the number of elements to be weighted. The statistical study shows that, whatever the notation scale, his indicator strongly decreases when the number of elements to be weighted increases.
} 


\subsection{Bryson and Joseph's indicator}

Bryson and Joseph's method [5] formulates the mapping from a non necessarily reciprocal vote cube $C$ to a suitable set of weights in a mathematical programming approach. Since there is no need to limit the number of entries per binary comparison, no preliminary synthesis of the individual judgments is required. Up to a logarithmic transformation, their approach is presented in formula 3.

$$
\mid \begin{array}{l:c}
\text { C.I. Bryson and Joseph } & =\frac{1}{\min \left(\prod_{k=1}^{d} \prod_{i=1}^{n} \prod_{j=1}^{n} p_{i j k} q_{i j k}\right)^{\frac{1}{d \times n \times(n-1)}}}, \\
\operatorname{with} & \frac{w_{i}}{w_{j}} \times \frac{p_{i j k}}{q_{i j k}}=c_{i j k} \quad i, j=1,2, \ldots, n ; k=1,2, \ldots, d .
\end{array}
$$

In the associated constraints each ratio of weights $w_{i} / w_{j}$ is multiplied by a ratio of real numbers $p_{i j k} / q_{i j k}\left(p_{i j k} \geq 1\right.$ and $q_{i j k}=1$ or $p_{i j k}=1$ and $\left.q_{i j k} \geq 1\right)$ in order to coincide with the vote $c_{i j k}$ of decision maker $k$. The geometric mean of the products $p_{i j k} q_{i j k}$ constitutes the objective function to minimize. The optimum represents the minimum average value that each entry in the vote cube would have to be multiplied or divided by in order to reach consistency. The inverse of this quantity has been chosen as consistency indicator by Bryson and Joseph. It ranges between 0 (maximal inconsistency) and 1 (perfect consistency). In their illustrative example, Bryson and Joseph adopt an arbitrary cut-off value (80\%) and assess the consistency of both the group and the individual DMs.

\section{An indicator based on the logarithmic least squares regression approach}

\subsection{The linear regression approach}

A regression model may be considered as an optimized approximation of the relation between a random variable said to be dependent and a set of prediction variables assumed not to be random.

In linear regression [8], an observation $Y_{i}$ of the dependent variable $y$ is related to values taken by prediction variables $x_{1}, \ldots, x_{n}$ through equations of the form $Y_{i}=\eta_{0}+\eta_{1} X_{1, i}+\ldots+\eta_{n} X_{n, i}+\varepsilon_{i}{ }^{7}$. The constant coefficients $\eta_{0}, \eta_{1}, \ldots \eta_{n}$ are parameters to be estimated for completing the model. Coefficient $\varepsilon_{i}$ is a random coefficient of error representing the difference between the linear model's estimation and the observation $i$. In matricial notation, the equation set may be expressed as:

\footnotetext{
${ }^{7}$ For example, if one assumes that the size $S$ of a person (dependent variable) is linearly related to his or her age $A$ (prediction variable), a linear regression approach consists in finding the straight line, of the form $S_{i}=\eta_{0}+\eta_{1} A_{i}$, modeling at best a set of (age, size) measurements performed on a sample of representative individuals.
} 


$$
Y=X H+E \text {, with } Y=\left(\begin{array}{c}
Y_{1} \\
Y_{2} \\
\vdots \\
Y_{m}
\end{array}\right), X=\left(\begin{array}{cccc}
X_{1,1} & X_{1,2} & \ldots & X_{1, n} \\
X_{2,1} & X_{2,2} & \ldots & X_{2, n} \\
\vdots & \vdots & \ddots & \vdots \\
X_{m, 1} & X_{m, 2} & \ldots & X_{m, n}
\end{array}\right), H=\left(\begin{array}{c}
\eta_{1} \\
\eta_{2} \\
\vdots \\
\eta_{n}
\end{array}\right), E=\left(\begin{array}{c}
\varepsilon_{1} \\
\varepsilon_{2} \\
\vdots \\
\varepsilon_{m}
\end{array}\right) \text {. }
$$

In this framework, the least squares estimator of $H$, represented in the following by $\Theta$, corresponds to the minimal sum of error squares $\sum\left(Y_{i}-\hat{Y}_{i}\right)^{2}$ between the measured values of the dependent variable $(Y)$ and the estimated ones $(\hat{Y}=X \Theta)$. When $\Theta$ exists it is the solution of the normal equation set: $X^{t} Y=X^{t} X \Theta$. If the errors follow a probabilistic distribution with a mean equal to zero, $\Theta$ is considered as a non biased estimator of $H([7]$, [8]).

\subsection{Application to the pairwise comparison framework}

In order to discuss the application of the linear regression approach to the context of pairwise comparison, let us first, introduce the binary parameter $\alpha_{i j k}$

$$
\alpha_{i j k}=\left\{\begin{array}{l}
1, \text { if DM } k \text { expresses an opinion on comparison } c_{i j}, \\
0, \text { otherwise and } c_{i j k}=c_{0}>0 \text { (arbitrary positive constant). }
\end{array}\right.
$$

If one considers $Y_{i j k}=\alpha_{i j k} \log \left(c_{i j k}\right) i, j=1, \ldots, n, \quad i \neq j$ as the observations of the dependent variable, the equation $\alpha_{i j k} \log \left(c_{i j k}\right)=\alpha_{i j k}\left(\log \left(w_{i}\right)-\log \left(w_{j}\right)+\varepsilon_{i j k}\right)^{8}$ links in a linear manner $Y_{i j k}$ to the set of prediction variables:

$$
\left(X_{i j k, l}=0, \ldots, X_{i j k, i}=\alpha_{i j k}, X_{i j k, i+1}=0, \ldots, X_{i j k, j}=-\alpha_{i j k}, X_{i j k, j+l}=0, \ldots, X_{i j k, n}=0\right),
$$

with the constant coefficients to be estimated:

$$
\left(\eta_{0}=0, \eta_{1}=\log \left(w_{1}\right), \ldots, \eta_{i}=\log \left(w_{i}\right), \ldots, \eta_{j}=\log \left(w_{j}\right), \ldots, \eta_{n}=\log \left(w_{n}\right)\right) \text {. }
$$

Via this logarithmic transformation, the linear regression has been applied to the pairwise comparison field since the eighties by De Graan [9] and Lootsma [15], in order to handle incomplete comparison matrices ${ }^{9}$. As illustrated in [14], this technique called Logarithmic Least Squares Regression (LLSR) generalizes the geometric mean based pairwise comparison approach and provides, at least from a probabilistic point of view, a pertinent estimator of the weights' vector [7].

\subsection{Consistency indicator}

In a regression model, the estimated sum of squares is always lower than the sum of squares of the observations. The difference, called residual sum of squares or errors sum of squares, corresponds to the information the model is unable to explain. This is a classical result in the literature on regression (see for example [8]). A short proof is given in formula 4.

\footnotetext{
${ }^{8}$ With equations of the type $\alpha_{i j k} \log \left(c_{i j k}\right)=\alpha_{i j k}\left(\log \left(w_{i}\right)-\log \left(w_{j}\right)+\varepsilon_{i j k}\right)$, the regression approach is unable to represent the reflexive opinions $\left(c_{i i k}, i=1,2, \ldots, n ; k=1,2, \ldots, d\right)$ which are assumed equal to 1 . This assumption does not deteriorate the quality of the optimal weight vector and the induced comparison matrix is reciprocal by construction.

${ }^{9}$ The lack of opinions can be tolerated so long the rank of the normal equations system remains equal to $n-1$. To fulfill this condition each element to rate/weight must be represented in at least one opinion and no pair of elements $\left(e_{i}, e_{j}\right)$ must be disjointed by transitivity (i.e. $\left.\alpha_{i h k} \alpha_{h j k}=0, h=1,2, \ldots, n ; k=1,2, \ldots, d\right)$.
} 


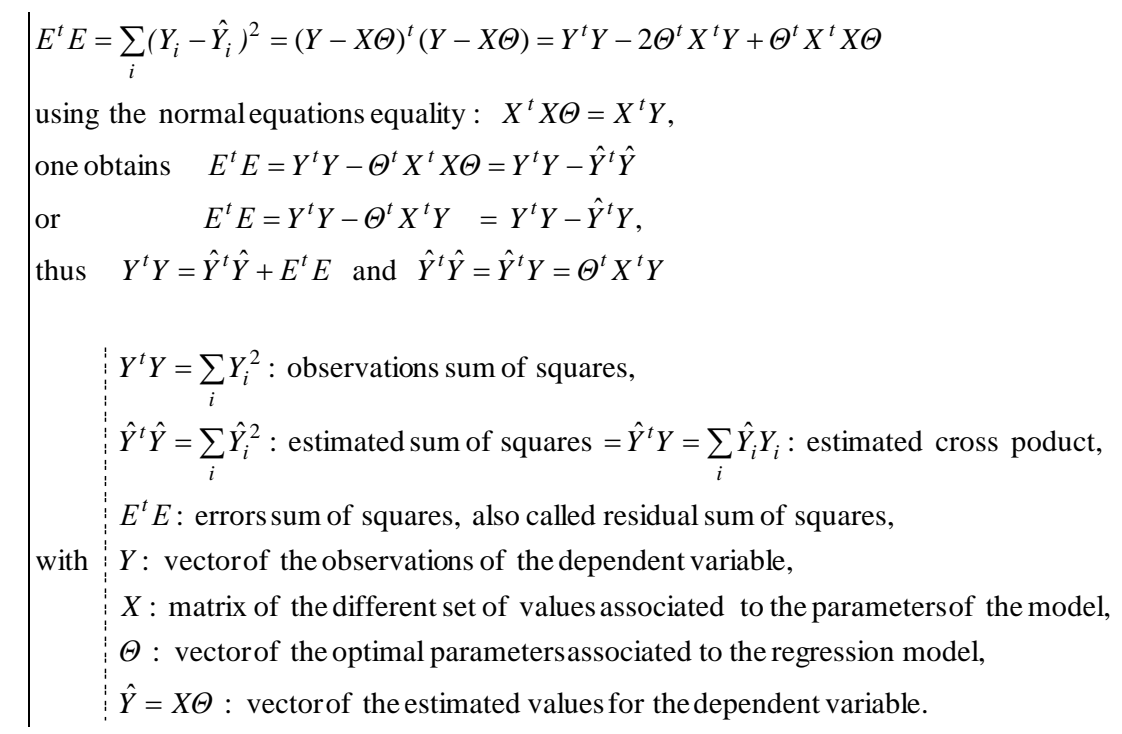

The ratio: (estimated sum of squares) / (sum of squares of the observations) is given by: $\left(\hat{Y}^{t} \hat{Y} / Y^{t} Y\right)=\left(\Theta^{t} X^{t} Y / Y^{t} Y\right)$. This quantity is an alternative form of the well known $R^{2}$ determination factor for models with no constant term ${ }^{10}$. The ratio represents the Explained Fraction of the Observation sum of Squares. In this paper, it is named EFOS to avoid confusion with the canonical form of the $R^{2}$ determination factor. For reciprocal comparison cubes, EFOS' value is identical to the $R^{2}$ determination factor and expresses the fraction of the original variance explained by the regression model.

In the context of pairwise comparisons this ratio constitutes a consistency indicator since it measures the adequacy between the consistent matrix induced by the estimated weights and the original comparison cube ${ }^{11}$. EFOS ranges between 0 when the original comparison cube is extremely inconsistent and 1 when estimations and observations fully coincide. The more consistent the original cube, the higher the explicative power of the LLSR model.

In the following section, EFOS is extended towards a more selective assessment of inconsistency in order to take several vote strategies into account within a group decision-making framework.

\section{Selective assessment of inconsistency}

As illustrated in section 1, the notion of group inconsistency should not be restricted to the notion of cardinal or ordinal inconsistency. More generally, indicators should be able to reflect the rules underlying the vote strategy and point out the inconsistency that the decision group is willing to correct.

\footnotetext{
${ }^{10}$ Typically, the $R^{2}$ correlation factor requires the presence of an $\eta_{0}$ among the parameters of the model before optimization. However, in the regression model associated to the general pairwise comparison problem, there is no $\eta_{0}$ parameter: $\eta_{0}$ is, intrinsically, set to 0 . The addition of an $\eta_{0}$ parameter is only possible if one assumes the vote cube to be reciprocal. In such a case the least squares optimum corresponds to $\eta_{0}=0$.

${ }^{11}$ Crawford and Williams' indicator [7] is also inspired from the LLSR approach. They suggested the use of the residual mean square to measure consistency. This quantity ( $E^{t} E /$ number of degrees of freedom) is an unbiased estimator of the variance of the errors. Aguarón and Moreno- Jiménez [1] showed a linear correlation between Crawford and Williams' indicator and Saaty's one.
} 


\subsection{A selective indicator}

In this section, the denominator of the indicator $\operatorname{EFOS}\left(Y^{t} Y=\Theta^{t} X^{t} Y+E^{t} E\right)$ is adjusted by relaxing from the error sum of squares $\left(E^{t} E\right)$ quantities that are tolerated and that should not be part of the information the model is expected to explain (as showed hereafter by formula 5).

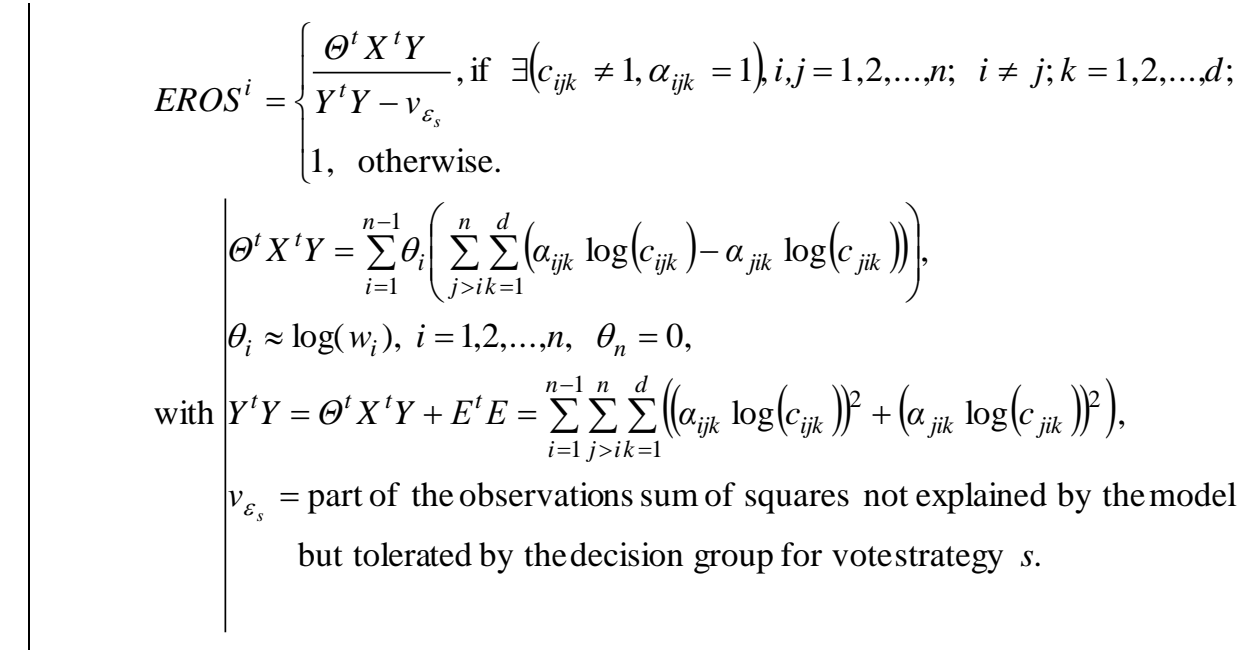

\subsection{Relaxed errors}

In the context of pairwise comparisons, the presence of different opinions for a same binary comparison $c_{i j}$ is a non avoidable source of errors which are called pure errors. At best any LLSR model will predict the average value $Y^{*}=\left(\sum_{k} Y_{i j k}\right) /\left(\sum_{k} \alpha_{i j k}\right)$.

In contrast, the presence of non reciprocal opinions $\left(\alpha_{i j x} \times c_{i j x} \neq \alpha_{j i y} \times 1 / c_{j i y}\right.$; $i, j=1,2, \ldots, n ; x, y=1,2, \ldots, d)$ generates fitting errors. For such symmetrical opinions, the best a linear model of the form $\hat{y}=x_{1}-x_{2}{ }^{12}$ can locally predict (ignoring the opinions of other DMs $)$ is $Y_{i j}{ }^{*}=\left(Y_{i j k}-Y_{j i k}\right) / 2$ and $Y_{j i}{ }^{*}=-\left(Y_{i j k}-Y_{j i k}\right) / 2=-Y_{i j}{ }^{*}$. Figures 2 and 3 illustrate the concept of pure and fitting errors ${ }^{13}$.

Based on such local reasonings, several alternatives are proposed for the quantity $v_{\varepsilon}$ by formula 6 . They represent errors that the linear model is enable to explain and that will be partly relaxed from the observations' sum of squares each time they contribute to inconsistencies the decision group tolerates.

\footnotetext{
${ }^{12}$ In the pairwise comparison framework the logarithmic transform of the estimated binary comparison $\hat{c}_{i j}$ is linked to the logarithmic transforms of the weights by the linear equation: $\log \left(\hat{c}_{i j}\right)=\log \left(w_{i}\right)-\log \left(w_{j}\right)$.

${ }^{13}$ See [16] for a detailed presentation of the concept of pure errors and fitting errors.
} 


$$
\begin{aligned}
& v_{\varepsilon 0}=0 \\
& v_{\varepsilon 1}=\sum_{i=1}^{n-1} \sum_{j \neq i}^{n} \sum_{j=1}^{d}\left(\alpha_{i j k}\left(\log \left(c_{i j k}\right)-m_{(i, j)}\right)^{2}\right) \\
& v_{\varepsilon 2}=\sum_{i=1}^{n-1} \sum_{j \neq i}^{n} \sum_{k=1}^{d}\left(\alpha_{i j k}\left(\log \left(c_{i j k}\right)-m_{(i, j), k}\right)^{2}\right) \\
& v_{\varepsilon 3}=\sum_{i=1}^{n} \sum_{j \neq i}^{n} \sum_{k=1}^{d}\left(\alpha_{i j k}\left(\log \left(c_{i j k}\right)-m_{(i, j), \neq k}\right)^{2}\right)
\end{aligned}
$$

Each $v_{\varepsilon_{i}}$ is computed as a sum of squared logarithmic differences between the considered opinions and local averages. These averages represent the LLSR model estimation when a subset of the opinions (constraints) is considered. For a given $v_{\varepsilon_{i}}$, the considered subsets of opinions are disjointed, which justifies an additive aggregation of the different squared differences.

- $m_{(i, j)}$ : logarithmic average expressing the absolute value of the LLSR's local estimation for all the opinions concerning the comparison $c_{i j}$ and its symmetrical $c_{j i}$.

$-m_{(i, j), k}:$ logarithmic average expressing the absolute value of the LLSR's local estimation for the opinion $c_{i j k}$ and its symmetrical $c_{j i k}$.

$-m_{(i, j), \nexists k}: \log$ arithmic average expressing the absolute value of the LLSR's local estimation for the set of opinions corresponding to the comparison $c_{i j}$ and its symmetrical $c_{j i}$ except the opinion $c_{j i k}$ replaced by $1 / c_{i j k}$. With this substitution, when $c_{j i k} \neq 1 / c_{i j k}$, the quantity $v_{\varepsilon_{3}}$ deduced from the sum of squares is smaller than $v_{\varepsilon_{1}}$, the one produced with $m_{(i, j)}$ (see the proof in the appendix). This procedure helps pointing out eventual inconsistencies on $c_{j i k}$.

$$
\text { with }\left\{\begin{array}{l}
d_{i j}=\sum_{k=1}^{d} \alpha_{i j k}: \text { number of opinions expressed on the binary comparison } c_{i j} . \\
m_{(i, j), k}=-m_{(j, i), k}=\left\{\begin{array}{l}
\frac{\alpha_{i j k} \times \log \left(c_{i j k}\right)-\alpha_{j i k} \times \log \left(c_{j i k}\right)}{\alpha_{i j k}+\alpha_{j i k}}, \text { if } \alpha_{i j k}+\alpha_{j i k}>0 \\
0, \text { otherwise. }
\end{array}\right. \\
m_{(i, j)}=-m_{(j, i)}=\left\{\begin{array}{l}
\sum_{l=1}^{d}\left(\alpha_{i j l} \times \log \left(c_{i j l}\right)-\alpha_{j i l} \times \log \left(c_{j i l}\right)\right) \\
d_{i j}+d_{j i}
\end{array}=\frac{\sum_{l=1}^{d}\left(m_{(i, j), l} \times\left(\alpha_{i j l}+\alpha_{j i l}\right)\right)}{d_{i j}+d_{j i}}, \text { if } d_{i j}+d_{j i}>0\right. \\
m_{(i, j), \neq k}=\left\{\begin{array}{l}
m_{(i, j)}+\left(\frac{\alpha_{j i k} \times \log \left(c_{i j k}\right)}{d_{i j}+d_{j i}}\right)+\left(\frac{\alpha_{j i k} \times \log \left(c_{j i k}\right)}{d_{i j}+d_{j i}}\right), \text { if } d_{i j}+d_{j i}>0 \\
0, \text { otherwise. }
\end{array}\right. \\
\text { for } i, j=1,2, \ldots, n ; i \neq j ; k=1,2, \ldots, d .
\end{array}\right.
$$

The local reasonings supporting the deduced part of errors $v_{\varepsilon_{i}}$ are schematically illustrated in figure 3. For the points representing the opinions of $\mathrm{DM}_{2}$, the global optimum (black line) induces higher fitting errors than the local optimum represented by the gray line.

It is easy to demonstrate that $v_{\varepsilon 1} \geq v_{\varepsilon 2}$ and $v_{\varepsilon l} \geq v_{\varepsilon 3}$ (see appendix). If the comparison cube is reciprocal $\left(\alpha_{i j x} c_{i j x}=\alpha_{j i y} / c_{j i y} ; i, j=1,2, \ldots, n ; x, y=1,2, \ldots, d\right)$, one gets the following equalities: $v_{\varepsilon 2}=v_{\varepsilon 0}=0$ and $v_{\varepsilon 3}=v_{\varepsilon 1}$ (in this case $v_{\varepsilon 3} \geq v_{\varepsilon 2}$ ). If the comparison cube reduces to a matrix ${ }^{14}$ (maximum one opinion per binary comparison), one gets $v_{\varepsilon 2}=v_{\varepsilon 1}$ and $v_{\varepsilon 3}=v_{\varepsilon 0}=0$ (in this case $v_{\varepsilon 2} \geq v_{\varepsilon 3}$ ). If the comparison cube reduces to a reciprocal matrix, one gets $v_{\varepsilon 3}=v_{\varepsilon 2}=v_{\varepsilon l}=v_{\varepsilon 0}=0$.

\footnotetext{
${ }^{14}$ For example, in a group where each DM is associated to a disjointed set of binary comparisons, the group's comparison cube reduces to a matrix.
} 
The part of errors $v_{\varepsilon_{1}}, v_{\varepsilon_{2}}$ and $v_{\varepsilon_{3}}$ are not disjointed since they involve common subsets of opinions. For this reason, it does not make sense to combine them additively. They express three different vote strategies presented in the following section.

\subsection{Implications in terms of tolerated inconsistencies}

For the group:

under $v_{\varepsilon 0}$, no specific inconsistency is tolerated;

$>$ under $v_{\varepsilon l}$, the decision group tolerates all inconsistencies induced by multiple or non reciprocal opinions on a binary comparison and its symmetrical $\left(c_{i j}\right.$ and $\left.c_{j i}\right)$;

$>$ under $v_{\varepsilon 2}$, the decision group tolerates only fitting errors relative to non reciprocal opinions expressed by a same DM $k$ (when $\alpha_{i j k} \times c_{i j k} \neq \alpha_{j i k} \times 1 / c_{j i k}$ );

$>$ under $v_{\varepsilon 3}$ the decision group considers (like for $v_{\varepsilon 1}$ ) each binary comparison and its symmetrical $\left(c_{i j}\right.$ and $\left.c_{j i}\right)$. In contrast with $v_{\varepsilon 2}$, it tolerates all inconsistencies induced by multiple or non reciprocal opinions except fitting errors caused by non reciprocal opinions expressed by a same DM $k$.

At individual DMs level (comparison matrices):

$>v_{\varepsilon 2}$ as $v_{\varepsilon 1}\left(v_{\varepsilon 2}=v_{\varepsilon 1}\right)$ expresses a tolerance towards inconsistent symmetrical opinions,

while, $v_{\varepsilon 3}$ as $v_{\varepsilon 0}\left(v_{\varepsilon 3}=v_{\varepsilon 0}=0\right)$ expresses a full consistency expectation.

\subsection{Limitations and perspectives}

The selective power of EFOS covers two basic situations: the non reciprocity of the symmetrical comparisons $\left(c_{i j} c_{j i} \neq 1\right)$ and the inconsistencies induced by the presence of multiple opinions per binary comparison $\left(c_{i j x} \neq c_{i j y}\right)$. For non reciprocal comparison cubes, EFOS does not take into account the notion of "indirect intransitivity" induced by paths of length $\geq 2$ (see note 4 ). Moreover, because it is based on local reasonings (see section 4.2), EFOS relaxes only partially the tolerated inconsistencies.

In addition, as any indicator using the regression approach (ex: [7]), EFOS does not consider the consistency of the diagonal comparisons (see note 8). The consistency of the diagonal comparisons can be of interest for some blind testing procedures.

Despite these limitations, EFOS is still able to qualitatively adjust its response to different vote strategies and different levels within the group structure.

\subsection{Applicative field}

To illustrate the applicative aspect of these different alternatives let us consider a blind test involving binary comparisons where the ordering for considering each of the two elements can significantly influence the result. This is true for sensorial evaluations as for example food and beverage tasting. In such a case it is quite normal to expect from a same DM non reciprocal opinions $\left(\alpha_{i j k} \times c_{i j k} \neq \alpha_{j i k} \times 1 / c_{j i k}\right)$, especially if the decision group includes non experts.

If, in addition, the decision group is willing to figure out the diversity of opinions (not a common opinion strategy), different appreciations should be tolerated for a given binary comparison $\left(c_{i j}\right)$. Under such conditions, $v_{\varepsilon l}$ is the alternative to choose. 
On the contrary, if the decision group seeks one common opinion (identical votes), it is important for the consistency indicator to point out inconsistencies due to non convergent opinions in the perspective of a compromise. In such case, $v_{\varepsilon 2}$ is the option to choose.

For a different context, where binary comparisons are not sensitive to the ordering of elements to be compared or where DMs' expertise is high, inconsistencies induced by non reciprocal opinions expressed by a same DM are less acceptable.

If the decision group's strategy is not common opinion oriented, $v_{\varepsilon 3}$ is the alternative to choose.

Otherwise, the coefficient $v_{\varepsilon 0}$ must be preferred.

Tables 2 and 3 respectively summarize the different strategies mentioned above, at both group and individual DMs' levels.

\begin{tabular}{|c|c|c|}
\cline { 2 - 3 } \multicolumn{1}{c|}{} & Uniform vote & Non uniform vote \\
\hline $\begin{array}{c}\text { Blind testing or } \\
\text { Low expertise }\end{array}$ & $v_{\varepsilon 2}$ & $v_{\varepsilon 1}$ \\
\hline High expertise & $v_{\varepsilon 0}$ & $v_{\varepsilon 3}$ \\
\hline
\end{tabular}

Tab. 2: Correspondence between the $v_{\varepsilon i}$ factors and group's voting strategies

\begin{tabular}{|c|c|}
\hline No tolerance & Tolerance for symmetrical inconsistencies \\
\hline$v_{\varepsilon 3}=v_{\varepsilon 0}$ & $v_{\varepsilon 2}=v_{\varepsilon 1}$ \\
\hline
\end{tabular}

Tab. 3: Correspondence between the $v_{\varepsilon i}$ factors and DMs voting strategies

\section{Illustrative example}

The following example consists in a blind quality test of three different olive oils. This example has been constructed in order to illustrate the consistency indicator and the different scenarios presented in the previous sections.

The test is achieved by 2 DMs invited to taste all possible combinations of different olive oils in different orders and without revealing samples' identity. When available, the estimations of the ratios $c_{i j}$ are expressed through the following qualitative rating scale: $\{4-$ : extremely week; $3-$ : very week; $2-$ : week; - : moderately week; $=$ : equal; + : moderately strong; $2+:$ strong; $3+$ : very strong; $4+$ : extremely strong $\}$. The comparison matrices are presented in table 4. 
DM1

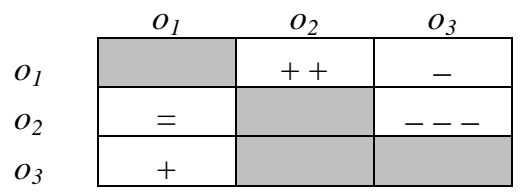

DM2

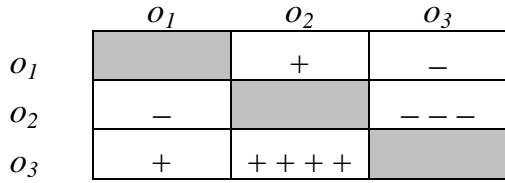

Tab. 4: DMs' comparison matrices

Table 5 summarizes consistency and weights' estimations for the different vote strategies presented in this paper both at DMs and group's level. In order to illustrate the rating scale's effect [20], the qualitative rating levels have been numerically translated according to two different ratio scales, respectively inspired from Saaty's [18] and Lootsma's [16] ones:

$$
\text { Sc. } 1=\left\{\frac{1}{9}, \frac{1}{6}, \frac{1}{4}, \frac{1}{2}, 1,2,4,6,9\right\} \text { and Sc. } 2=\left\{\frac{1}{16}, \frac{1}{8}, \frac{1}{4}, \frac{1}{2}, 1,2,4,8,16\right\} .
$$

\begin{tabular}{|c|c|c|c|c|c|c|c|c|c|c|c|c|c|c|c|c|c|c|}
\hline & \multicolumn{6}{|c|}{ Group } & \multicolumn{6}{|c|}{ DM1 } & \multicolumn{6}{|c|}{ DM2 } \\
\hline$E F O S^{3}$ & \multicolumn{3}{|c|}{0.927} & \multicolumn{3}{|c|}{0.906} & \multirow{2}{*}{\multicolumn{3}{|c|}{0.829}} & \multirow{2}{*}{\multicolumn{3}{|c|}{0.833}} & \multirow{2}{*}{\multicolumn{3}{|c|}{0.967}} & \multirow{2}{*}{\multicolumn{3}{|c|}{0.931}} \\
\hline$E F O S^{0}$ & \multicolumn{3}{|c|}{0.911} & \multicolumn{3}{|c|}{0.891} & & & & & & & & & & & & \\
\hline$E F O S^{1}$ & & 0.977 & & \multirow{2}{*}{\multicolumn{3}{|c|}{$\begin{array}{l}0.948 \\
0.945\end{array}$}} & \multirow{2}{*}{\multicolumn{3}{|c|}{0.984}} & \multirow{2}{*}{\multicolumn{3}{|c|}{0.961}} & \multirow{2}{*}{\multicolumn{3}{|c|}{0.975}} & \multirow{2}{*}{\multicolumn{3}{|c|}{0.947}} \\
\hline$E F O S^{2}$ & \multicolumn{3}{|c|}{0.975} & & & & & & & & & & & & & & & \\
\hline & & Sc. 1 & & & Sc. 2 & & \multicolumn{3}{|c|}{ Sc. 1} & \multicolumn{3}{|c|}{ Sc. 2} & \multicolumn{3}{|c|}{ Sc. 1} & \multicolumn{3}{|c|}{ Sc. 2} \\
\hline & $w_{l}$ & $w_{2}$ & $w_{3}$ & $w_{1}$ & $w_{2}$ & $w_{3}$ & $w_{1}$ & $w_{2}$ & $w_{3}$ & $w_{l}$ & $w_{2}$ & $w_{3}$ & $w_{l}$ & $w_{2}$ & $w_{3}$ & $w_{1}$ & $w_{2}$ & $w_{3}$ \\
\hline & 26.5 & 11.2 & 62.3 & 24.9 & 9.4 & 65.7 & 27.3 & 12.3 & $60.4 \vdots$ & 26.3 & 11.1 & 62.6 & 25.9 & 10.6 & 63.5 & 23.9 & 8.5 & 67.6 \\
\hline
\end{tabular}

Tab. 5: Results for the different vote strategies

For the two alternative scales, the numerical results confirm the incidence of the selective assessment of inconsistency and the ability of EFOS to handle the four vote strategies detailed in section 4.5. As expected the relaxed variants of the indicator (indexes $\{1,2,3\}$ ) provide higher consistency rates ${ }^{15}$. At DMs' level, the differences between selective versions and non selective ones are higher for DM1 who has provided a more inconsistent comparison matrix and has taken bigger advantage of the relaxation of reciprocal inconsistencies. Furthermore, this example illustrates the ability of the indicator to tackle incomplete comparison matrices and cubes.

In order to compare EFOS to the two other indicators dedicated to group decision making, presented in the literature review, the previous comparison matrices of table 4 has been transformed into symmetrical ones by keeping unchanged the upper triangular half-matrices.

\footnotetext{
${ }^{15}$ Theoretically EFOS ${ }^{1}$ refers to more local optimums and thus relaxes higher sums of errors than $E F O S^{2}$ or $E F O S^{3}$ (see the proof in the appendix).
} 


\begin{tabular}{|c|c|c|c|c|c|c|c|c|c|c|c|c|c|c|c|c|c|c|}
\hline & \multicolumn{6}{|c|}{ Group } & \multicolumn{6}{|c|}{ DM1 } & \multicolumn{6}{|c|}{ DM2 } \\
\hline$E F O S^{0}$ & \multirow{2}{*}{\multicolumn{3}{|c|}{0.975}} & \multirow{2}{*}{\multicolumn{3}{|c|}{0.973}} & \multirow{4}{*}{\multicolumn{3}{|c|}{0.995}} & \multirow{4}{*}{\multicolumn{3}{|c|}{1.000}} & \multirow{4}{*}{\multicolumn{3}{|c|}{0.987}} & \multirow{4}{*}{\multicolumn{3}{|c|}{0.970}} \\
\hline$E F O S^{2}$ & & & & & & & & & & & & & & & & & & \\
\hline EFOS ${ }^{1}$ & \multirow{2}{*}{\multicolumn{3}{|c|}{1.000}} & \multirow{2}{*}{\multicolumn{3}{|c|}{0.993}} & & & & & & & & & & & & \\
\hline$E F O S^{3}$ & & & & & & & & & & & & & & & & & & \\
\hline$C I_{\text {Saaty }}$ & \multirow{2}{*}{\multicolumn{3}{|c|}{0.0001}} & \multirow{2}{*}{\multicolumn{3}{|c|}{0.0045}} & \multicolumn{3}{|c|}{0.0031} & \multicolumn{3}{|c|}{0.0000} & \multicolumn{3}{|c|}{0.0061} & \multicolumn{3}{|c|}{0.0179} \\
\hline$C I_{\text {Saatv DM/group }}$ & & & & & & & \multicolumn{3}{|c|}{0.0121} & \multicolumn{3}{|c|}{0.0089} & \multicolumn{3}{|c|}{0.0152} & \multicolumn{3}{|c|}{0.0271} \\
\hline \multirow[t]{3}{*}{$C I_{B r v s o n}$ and Joseph } & \multicolumn{3}{|c|}{0.891} & \multicolumn{3}{|c|}{0.889} & \multicolumn{3}{|c|}{0.909} & \multicolumn{3}{|c|}{1.000} & \multicolumn{3}{|c|}{0.874} & \multicolumn{3}{|c|}{0.794} \\
\hline & \multicolumn{3}{|c|}{ Sc. 1} & \multicolumn{3}{|c|}{ Sc. 2} & \multicolumn{3}{|c|}{ Sc. 1} & \multicolumn{3}{|c|}{ Sc. 2} & \multicolumn{3}{|c|}{ Sc. 1} & & Sc. 2 & \\
\hline & $w_{l}$ & $w_{2}$ & $w_{3}$ & $w_{l}$ & $w_{2}$ & $w_{3}$ & $w_{l}$ & $w_{2}$ & $w_{3}$ & $w_{l}$ & $w_{2}$ & $w_{3}$ & $w_{l}$ & $w_{2}$ & $w_{3}$ & $w_{l}$ & $w_{2}$ & $w_{3}$ \\
\hline$L L S R$ & 29.5 & 10.3 & 60.2 & 28.1 & 8.8 & 63.1 & 32.3 & 8.9 & 58.8 & 30.8 & 7.7 & 61.5 & 26.9 & 11.7 & 61.4 & 25.5 & 10.1 & 644 \\
\hline$E V$ & & & & & & & & & & & & & & & & & & \\
\hline$M P$ & 30 & 10 & 60 & 30.7 & 7.8 & 61.5 & 32.3 & 8.9 & 58.8 & 30.8 & 7.7 & 61.5 & 26.5 & 11.2 & 62.3 & 24.9 & 9.4 & 65.7 \\
\hline
\end{tabular}

Tab. 6: Results of the consistency indicators EFOS, $C I_{\text {Saaty }}$ and $C I_{\text {Bryson and Joseph }}$

Table 6 presents the different results, detailing the weights for the three methods: LLSR, EV ${ }^{16}$ and the MP model of Bryson and Joseph. Unlike Saaty's indicator that varies in the range $[0,+\infty$ [ to indicate a decreasing consistency, the two others indicates an increasing consistency from 0 to 1 .

Bryson and Joseph's indicator is the only one that restricts to the distinction between DM's level and group's level. In addition, Saaty's indicator is able to merge the two levels. It measures both the consistency and the deviation of DM's judgments from those of the group ${ }^{17}$. For such reciprocal comparison cubes, EFOS' selective power restricts to the inconsistency introduced by the multiplicity of the opinions. EFOS is still the only indicator that allows the distinction between several vote strategies (two in this case, as detailed in section 4.5).

\section{Conclusion}

The concept of selective assessment of consistency, introduced by this paper, is quite innovative and complementary of aspects addressed by previous approaches.

Compared to its benchmarks within a mono-criteria group decision making framework, the consistency indicator EFOS, introduced in this paper, has the advantage of delivering consistency measures that take several group's strategies into account.

Despite the fact that the selective power of EFOS is still partial, such a flexibility allows a more realistic modeling of pairwise comparisons in group decision making.

\footnotetext{
${ }^{16}$ For $n=3$ and a comparison matrix with exactly one opinion per comparison EV and LLSR produce equivalent weight vectors [7].

17 A similar approach could be applied with EFOS. This can be illustrated in formula 5 by considering the case of individual DMs and substituting the weights (in $\Theta$ ) by those of the group.
} 


\section{References}

[1] Aguarón J., Moreno-Jiménez J. M. (2003), The geometric consistency index: Approximated thresholds. European Journal of Operational Research, vol. (147): p.137-145. [2] Barzilai J., (1998), Measurement Foundations for Preference Function Modelling. IEEE International Conference on Systems, Man and Cybernetics, San Diego, California, October 1998.

[3] Belton V. and Pictet J., (1997), A Framework for Group Decision Using a MCDA Model : Sharing, Aggregating or Comparing Individual Information? Revue des systèmes de décision, vol. (6) - n³: p. 283 - 303.

[4] Boender C.G.E., de Graan J.G., Lootsma F.A., (1989), Multi-criteria decision analysis with fuzzy pairwise comparisons. Fuzzy Sets and Systems, vol. (29): p. 133 - 143.

[5] Bryson N.K.-M., Joseph A., (1999), Generating consensus priority point vectors: a logarithmic goal programming approach. Computers \& Operations Research, vol. (26): p. 637-643.

[6] Condon E., Golden B., Wasil E. (2003), Visualizing group decision in the analytic hierarchy process. Computers \& Operations Research, vol. (30): p. 1435-1445.

[7] Crawford G., Williams C., (1985), A Note on the Analysis of Subjective Judgments Matrices. Journal of Mathematical Psychology, vol. (29): p. 387-405.

[8] Draper N.R., Smith H., (1980), Applied Regression Analysis. second ed. Willey series in probability and mathematical statistics, ed. Sons J.W., New York.

[9] de Graan J.G., (1980), Extensions to the multiple criteria analysis of T. L. Saaty. Report National Institute of Water Supply.

[10] Escobar M.T., Moreno-Jiménez J.M., (2000), Reciprocal distributions in the analytic hierarchy process. European Journal of Operational Research, vol. (123): p. 154-174.

[11] Fechner G.T., Elements of Psychophysics. Vol. volume 1, Holt, Rinehart \& Winston, New-York, 1965; translation by H. E. Adler of Elemente der Psychophysik, Breitkopf und Härtel, Leipzig, (1860).

[12] Golden B.L., Wang Q., (1989), An alternate measure of consistency, in: The Analytic Hierarchy Process: Application and Studies. Golden B., Wasil E. Editors, Springer, New York, p. 68-81.

[13] Limayem F., (2001), Modèles de pondération par les méthodes de tri croisé pour l'aide à la décision collaborative en projet. $\mathrm{Ph}$. D. thesis, November $23^{\text {th }} 2001$, Ecole Centrale Paris. [14] Limayem F., Yannou B., (2004), Generalization of the RCGM and LSLR Pairwise Comparison Methods. Computes and Mathematics with Applications, vol. (48): p. 539-548. [15] Lootsma F.A., (1982), Performance evaluation of nonlinear optimization methods via multi-criteria decision analysis and via linear model analysis, in Nonlinear Optimization, Powell M.J.D. Editor, Academic Press: London, vol. (1): p. 419-453.

[16] Lootsma F. A., (1996), A model for the relative importance of the criteria in the multiplicative AHP and SMART. European Journal of Operational Research, vol. (94): p. 467476.

[17] Roy B. and Mousseau V., (1996), A theoretical framework for Analysing the Notion of Relative Importance of Criteria. Journal of Multi-Criteria Decision Analysis, vol. (5): p. 145159.

[18] Saaty T.L., (1977), A scaling method for priorities in hierarchical structures. Journal of Mathematical Psychology, vol. 15(3): p. 234-281.

[19] Saaty T.L., (1989) Group decision-making and the AHP, in: The Analytic Hierarchy Process: Applications and Studies. Golden B., Wasil E. Editors, Springer, New York, p. 5967.

[20] Salo A. A. and Hämäläinen R. P., (1997), On the Measurement of Preferences in the Analytic Hierarchy Process. Journal of Multi-Criteria Decision Analysis, vol. (6): p. 309-319. [21] Takeda E., (1992), A note on consistent adjustments of pairwise comparison judgments. Mathematical and Computer Modelling, vol. (17): p. 29-35. 


\section{Appendix}

$1-v_{\varepsilon l} \geq v_{\varepsilon 3}$

\section{Proof}

$*$ if $d_{i j}+d_{j i}>0$

Let $b_{i j k}=\log \left(c_{i j k}\right)$ and $t=\frac{\log \left(c_{i j k}\right)+\log \left(c_{j i k}\right)}{d_{i j}+d_{j i}}=\frac{b_{i j k}+b_{j i k}}{d_{i j}+d_{j i}}$.

$\varepsilon_{1}-\varepsilon_{3}=\sum_{i=1}^{n-1} \sum_{j \neq i}^{n} \sum_{k=1}^{d} \alpha_{i j k}\left(\left(b_{i j k}-m_{(i, j)}\right)^{2}-\left(b_{i j k}-m_{(i, j), \neq k}\right)^{2}\right)$

By using the equalitiy $m_{(i, j), \neq k}=m_{(i, j)}+\alpha_{j i k} t$, one obtains :

$$
\begin{aligned}
\varepsilon_{1}-\varepsilon_{3} & =\sum_{i=1}^{n-1} \sum_{j \neq i k=1}^{n} \sum_{i j k}^{d} \alpha_{i j k}\left(\left(b_{i j k}-m_{(i, j)}\right)^{2}-\left(b_{i j k}-m_{(i, j)}-\alpha_{j i k} t\right)^{2}\right) \\
& =\sum_{i=1}^{n-1} \sum_{j \neq i k=1}^{n} \sum_{i j k}^{d} \alpha_{i j k}\left(\left(b_{i j k}-m_{(i, j)}\right)^{2}-\left(b_{i j k}-m_{(i, j)}\right)^{2}-\alpha_{j i k}^{2} t^{2}+2 \alpha_{j i k} t\left(b_{i j k}-m_{(i, j)}\right)\right)
\end{aligned}
$$

By using the equalitiy $\alpha_{j i k}^{2}=\alpha_{j i k}$ and distiguish ing the cases $(j<i)$ and $(j>i)$, one obtains:

$$
\begin{aligned}
\varepsilon_{1}-\varepsilon_{3} & =\sum_{i=1}^{n-1} \sum_{j>i}^{n} \sum_{k=1}^{d}\left(\alpha_{i j k} \alpha_{j i k} t\left(\left[2\left(b_{i j k}-m_{(i, j)}\right)-t\right]+\left[2\left(b_{j i k}-m_{(j, i)}\right)-t\right]\right)\right) \\
& =\sum_{i=1}^{n-1} \sum_{j>i}^{n} \sum^{d}\left(2 \alpha_{i j k} \alpha_{j i k} t\left(b_{i j k}-m_{(i, j)}+b_{j i k}-m_{(j, i)}-t\right)\right)
\end{aligned}
$$

By using the equalities $m_{(i, j)}=-m_{(j, i)}$ and $b_{i j k}+b_{j i k}=t\left(d_{i j}+d_{j i}\right)$, one obtains:

$$
\begin{aligned}
\varepsilon_{1}-\varepsilon_{3} & =\sum_{i=1}^{n-1} \sum_{j>i}^{n} \sum_{k=1}^{d}\left(2 \alpha_{i j k} \alpha_{j i k} t\left(b_{i j k}+b_{j i k}-t\right)\right) \\
& =\sum_{i=1}^{n-1} \sum_{j>i}^{n} \sum_{k=1}^{d}\left(2 \alpha_{i j k} \alpha_{j i k} t^{2}\left(d_{i j}+d_{j i}-1\right)\right) \geq 0 .
\end{aligned}
$$

$*$ if $d_{i j}+d_{j i}=0, \quad \varepsilon_{1}=\varepsilon_{3}=0$.

$2-v_{\varepsilon 1} \geq v_{\varepsilon 2}$

\section{Proof}

$*$ if $d_{i j}+d_{j i}>0$,

Let $b_{i j k}=\log \left(c_{i j k}\right)$, by distiguish ing the cases $(j<i)$ and $(j>i)$, and using the equalities $m_{(i, j)}=-m_{(j, i)}$ and $m_{(i, j), k}=-m_{(j, i), k}$, one obtains :

$$
\begin{aligned}
\varepsilon_{1}-\varepsilon_{2}= & \sum_{i=1}^{n-1} \sum_{j>i}^{n} \sum_{k=1}^{d}\left(\begin{array}{l}
\alpha_{i j k}\left(b_{i j k}-m_{(i, j)}\right)^{2}+\alpha_{j i k}\left(b_{j i k}-m_{(j, i)}\right)^{2} \\
-\alpha_{i j k}\left(b_{i j k}-m_{(i, j), k}\right)^{2}-\alpha_{j i k}\left(b_{j i k}-m_{(j, i), k}\right)^{2}
\end{array}\right) \\
= & \left.\sum_{i=1}^{n-1} \sum_{j>i}^{n} \sum_{j=1}^{d}\left(\begin{array}{l}
\alpha_{i j k}\left(b_{i j k}^{2}-2 b_{i j k} m_{(i, j)}+m_{(i, j)}^{2}\right)+\alpha_{j i k}\left(b_{j i k}^{2}-2 b_{j i k} m_{(j, i)}+m_{(j, i)}^{2}\right) \\
-\alpha_{i j k}\left(b_{i j k}^{2}-2 b_{i j k} m_{(i, j), k}+m_{(i, j), k}^{2}\right)-\alpha_{j i k}\left(b_{j i k}^{2}-2 b_{j i k} m_{(j, i), k}+m_{(j, i), k}^{2}\right)
\end{array}\right)\right) \\
= & \sum_{i=1}^{n-1} \sum_{j>i k=1}^{n} \sum_{i=1}^{d}\left(\begin{array}{l}
m_{(i, j)}^{2}\left(\alpha_{i j k}+\alpha_{j i k}\right)-2 m_{(i, j)}\left(\alpha_{i j k} b_{i j k}-\alpha_{j i k} b_{j i k}\right) \\
-m_{(i, j), k}^{2}\left(\alpha_{i j k}+\alpha_{j i k}\right)+2 m_{(i, j), k}\left(\alpha_{i j k} b_{i j k}-\alpha_{j i k} b_{j i k}\right)
\end{array}\right)
\end{aligned}
$$




$$
=\sum_{i=1}^{n-1} \sum_{j>i}^{n}\left(\begin{array}{l}
m_{(i, j)}^{2} \sum_{k=1}^{d}\left(\alpha_{i j k}+\alpha_{j i k}\right)-2 m_{(i, j)} \sum_{k=1}^{d}\left(\alpha_{i j k} b_{i j k}-\alpha_{j i k} b_{j i k}\right) \\
-\sum_{k=1}^{d} m_{(i, j), k}^{2}\left(\alpha_{i j k}+\alpha_{j i k}\right)+2 \sum_{k=1}^{d} m_{(i, j), k}\left(\alpha_{i j k} b_{i j k}-\alpha_{j i k} b_{j i k}\right)
\end{array}\right)
$$

By using the equalities : $d_{i j}=\sum_{k=1}^{d} \alpha_{i j k}, m_{(i, j)}=\frac{\sum_{k=1}^{d} m_{(i, j), k} \times\left(\alpha_{i j k}+\alpha_{j i k}\right)}{d_{i j}+d_{j i}}$

$$
\begin{aligned}
& \text { and } m_{(i, j), k}=\frac{\left(\alpha_{i j k} b_{i j k}-\alpha_{j i k} b_{j i k}\right)}{\alpha_{i j k}+\alpha_{j i k}} \text {, one obtains : } \\
& \varepsilon_{1}-\varepsilon_{2}=\sum_{i=1}^{n-1} \sum_{j>i}^{n}\left(\begin{array}{l}
m_{(i, j)}^{2}\left(d_{i j}+d_{j i}\right)-2 m_{(i, j)} \sum_{k=1}^{d} m_{(i, j), k}\left(\alpha_{i j k}+\alpha_{j i k}\right) \\
-\sum_{k=1}^{d} m_{(i, j), k}^{2}\left(\alpha_{i j k}+\alpha_{j i k}\right)+2 \sum_{k=1}^{d} m_{(i, j), k}^{2}\left(\alpha_{i j k}+\alpha_{j i k}\right)
\end{array}\right) \\
& =\sum_{i=1}^{n-1} \sum_{j>i}^{n}\left(m_{(i, j)}{ }^{2}\left(d_{i j}+d_{j i}\right)-2 m_{(i, j)}^{2}\left(d_{i j}+d_{j i}\right)+\sum_{k=1}^{d} m_{(i, j), k}^{2}\left(\alpha_{i j k}+\alpha_{j i k}\right)\right) \\
& =\sum_{i=1}^{n-1} \sum_{j>i}^{n}\left(-m_{(i, j)}^{2}\left(d_{i j}+d_{j i}\right)+\sum_{k=1}^{d} m_{(i, j), k}^{2}\left(\alpha_{i j k}+\alpha_{j i k}\right)\right) \\
& =\sum_{i=1}^{n-1} \sum_{j>i}^{n}\left(-m_{(i, j)}^{2}\left(d_{i j}+d_{j i}\right)+\sum_{\substack{k=1 \\
k \neq l \text { if } \alpha_{i j l}+\alpha_{j i l}=0}}^{d} \frac{m_{(i, j)}^{2}}{\alpha_{i j k}+\alpha_{j i k}}\left(d_{i j}+d_{j i}\right)^{2}\right) \\
& =\sum_{i=1}^{n-1} \sum_{j>i}^{n} m_{(i, j)}^{2}\left(d_{i j}+d_{j i}\right)\left(-1+\sum_{\substack{k=1 \\
k \neq l \text { if } \alpha_{i j l}+\alpha_{j i l}=0}}^{d} \frac{d_{i j}+d_{j i}}{\alpha_{i j k}+\alpha_{j i k}}\right) \geq 0
\end{aligned}
$$

since $d_{i j}+d_{j i}=\sum_{h=1}^{d}\left(\alpha_{i j h}+\alpha_{j i h}\right) \geq \alpha_{i j k}+\alpha_{j i k}, \forall k=1,2, \ldots, d$.

$*$ if $d_{i j}+d_{j i}=0, \quad \varepsilon_{1}=\varepsilon_{2}=0$.

\section{Vitae}

Frej Limayem received a M. Sc. (1995) in Industrial Engineering from Ecole Nationale d'Ingénieurs de Tunis, and received a Ph.D. (2001) in industrial engineering from Ecole Centrale Paris. His Ph.D. was directed by Dr. Bernard Yannou and focused on the use of weighting models, based on pairwise comparison methods, for collaborative decision aid in project. More generally, his research interests concern decision making in product design and development.

Dr Bernard Yannou is an assistant Professor of Industrial and Mechanical Engineering at the Laboratoire Génie Industriel of Ecole Centrale of Paris, France. He received a M. Sc. (1988) in Mechanical Engineering from Ecole Normale Supérieure of Cachan, and a second M. Sc. (1989) in Computer Science from Paris-6 University. He received a Ph. D. (1994) in Industrial Engineering from Ecole Normale Supérieure of Cachan. His research interests are centered on the preliminary stages of the product design: defining the design requirements, synthesizing product concepts, rapid assessment of product performances, preference aggregation of the product and the project performances for the supervision of the design process. 


\begin{tabular}{c|cccc} 
& $e_{1}$ & $e_{2}$ & $\ldots$ & $e_{n}$ \\
\hline$e_{1}$ & $\left(e_{1}, e_{1}\right)$ & $\left(e_{1}, e_{2}\right)$ & $\ldots$ & $\left(e_{1}, e_{n}\right)$ \\
$e_{2}$ & $\left(e_{2}, e_{1}\right)$ & $\left(e_{2}, e_{2}\right)$ & $\ldots$ & $\left(e_{2}, e_{n}\right)$ \\
$\vdots$ & $\vdots$ & $\vdots$ & $\ddots$ & $\vdots$ \\
$e_{n}$ & $\left(e_{n}, e_{1}\right)$ & $\left(e_{n}, e_{2}\right)$ & $\ldots$ & $\left(e_{n}, e_{n}\right)$
\end{tabular}

Fig. 1. The comparison matrix

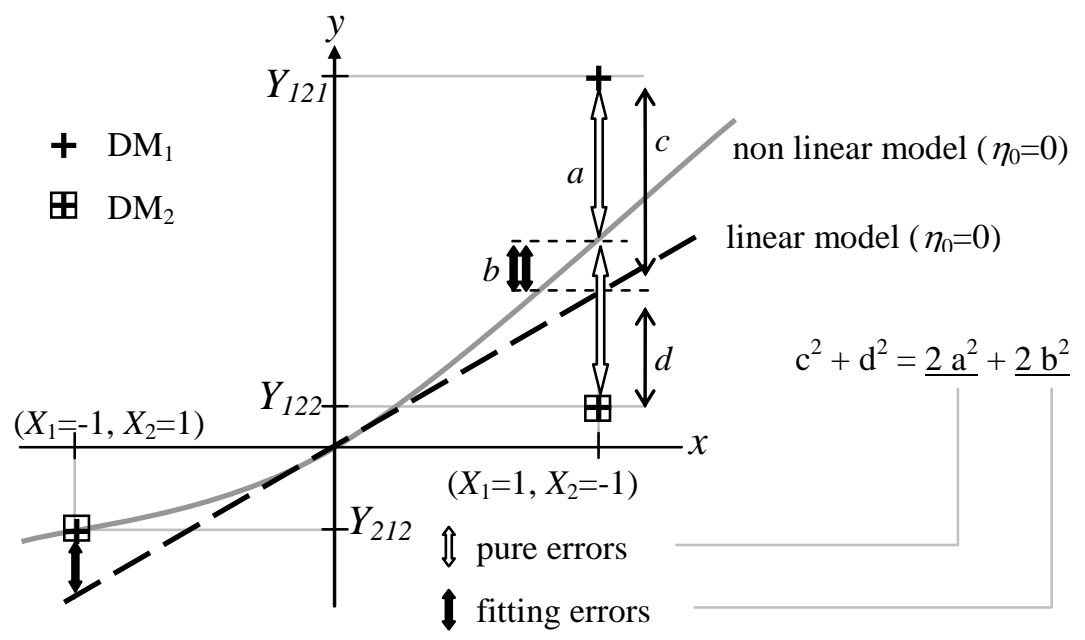

Fig. 2. Graphical illustration of pure and fitting errors on the case of a 2 × 2 comparison matrix where $Y_{i j k}$ represents the logarithm of the opinion of DM $k$ for the binary comparison $\left(e_{i}, e_{j}\right) .{ }^{181920}$

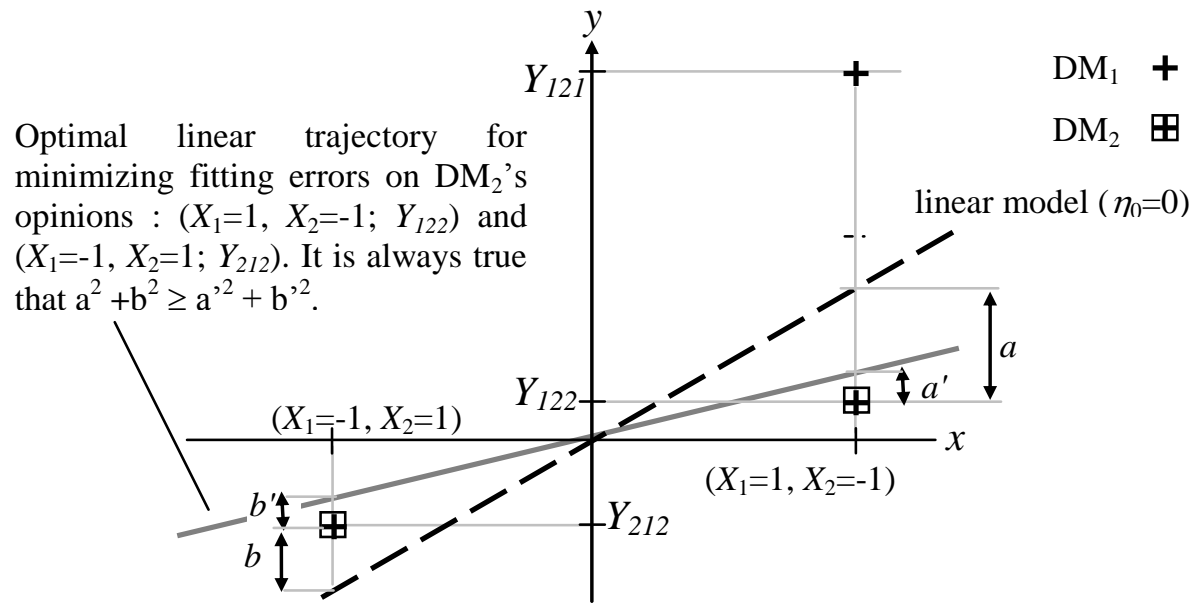

Fig. 3. Graphical illustration of the fact that local estimations generate less errors than global ones on the case of a $2 \times 2$ comparison matrix 1820

18 The $2 \times 2$ comparison matrix corresponds to a 2-variable-regression. The symmetry of the comparison matrix model allows a 2 dimensional representation on the plan $\left(x_{1}+x_{2}=0\right)$.

${ }^{19}$ Unlike the linear model the non linear one produces no fitting errors but none of them can avoid the pure errors. As illustrated by the equality $c^{2}+d^{2}=\underline{2 a^{2}}+\underline{2 b^{2}}$, the sum of errors' squares is equivalent to a sum of pure errors and fitting errors.

${ }^{20}$ In this example $\mathrm{DM}_{1}$ expresses no opinion on the binary comparison $\left(e_{2}, e_{1}\right)$. 